\title{
Bone metastasis predicts poor prognosis of patients with brain metastases from colorectal carcinoma post aggressive treatment
}

This article was published in the following Dove Press journal: Cancer Management and Research

\author{
Hao Duan ${ }^{1, *}$ \\ Zhen-Qiang $\mathrm{He}^{1, *}$ \\ Cheng-Cheng Guo' \\ Jue-Hui Li' \\ Jian Wang' \\ Zhe Zhu ${ }^{2}$ \\ Ke Sai' \\ Zhong-Ping Chen' \\ Xiao-Bing Jiang' \\ Yong-Gao Mou'
}

'Department of Neurosurgery/NeuroOncology, Sun Yat-sen University

Cancer Center, State Key Laboratory

of Oncology in South China,

Collaborative Innovation Center for

Cancer Medicine, Guangzhou, China;

${ }^{2}$ Department of Medicine, Division

of Regenerative Medicine, University

of California, San Diego, School of

Medicine, La Jolla, CA, USA

*These authors contributed equally to this work
Correspondence: Xiao-Bing Jiang;

Yong-Gao Mou

Department of Neurosurgery/Neuro-

Oncology, Sun Yat-sen University

Cancer Center, State Key Laboratory of Oncology in South China, Collaborative Innovation Center for Cancer Medicine, No. 65I, Dongfeng Road East, Guangzhou 510060, China

Tel +86 $2087343309 ;+862087343899$

Fax +862087343310

Email jiangxiaob I@sysucc.org.cn;

mouyg@sysucc.org.cn
Purpose: The presence of brain metastasis (BM) in patients with colorectal cancer (CRC) is usually associated with terminal-stage illness; however, a subgroup of patients receiving aggressive treatment can have a satisfactory prognosis. This study was designed to investigate the profile of prognostic factors in CRC patients with BM treated aggressively.

Patients and methods: CRC patients with BM were retrospectively reviewed. Survival analysis was performed to identify potential prognostic factors in the entire cohort of patients and a subgroup of patients treated aggressively. Aggressive treatments included surgical resection, radiotherapy, and/or chemotherapy. Overall survival was defined as the time between the diagnosis of BM and death or until the date of the last follow-up visit.

Results: A total of 78 CRC patients were confirmed as having BM. Sixty-eight of them had extracranial metastases at the time of their BM diagnosis. The most common sites of extracranial metastases were lung $(n=51,65.4 \%)$, followed by liver $(n=25,32.1 \%)$ and bone $(n=12,15.4 \%)$. Fifty-one patients who were treated aggressively had significantly longer overall survival than those who accepted palliative care ( 14.1 months vs 2.0 months, $P<0.0001)$. Multivariate analysis was applied, and the results showed that aggressive treatment $(\mathrm{n}=51)$, recursive partitioning analysis class $\mathrm{I} / \mathrm{II}$ (hazard ratio $[\mathrm{HR}]=0.27,95 \% \mathrm{CI}: 0.12-0.6, P=0.001$ ), and fewer BM (HR $=0.4,95 \%$ CI: $0.21-0.78, P=0.07$ ) predicted longer survival. In contrast, the presence of bone metastasis, rather than lung or liver metastasis, at the time of diagnosis of $B M(H R=2.38$, 95\% CI: $1.08-5.28, P=0.032$ ) predicted a poor prognosis.

Conclusions: Although the prognosis of CRC patients having BM is frequently very poor, those with good performance status and few brain lesions responded to aggressive treatment, while those with bone metastasis at the time of diagnosis of BM had relatively dismal survival rates, even when treated aggressively.

Keywords: brain metastasis, colorectal cancer, bone metastasis, aggressive treatment

\section{Introduction}

Colorectal cancer (CRC) represents one of the most frequent malignancies worldwide. ${ }^{1}$ Previous studies have shown that in many patients with CRC, metastasis usually appears earliest in liver and lung, followed by bone and brain. ${ }^{2-5}$ The risk of subsequent metastasis to the brain increases once CRC cells metastasize to the lung and bone. ${ }^{3}$ Moreover, CRC patients with limited involvement of lung or liver exhibit improved outcomes, whereas those with tumors with a predilection for spreading to the bone or brain tissue have worse outcomes. ${ }^{6}$ Therefore, CRC patients with brain metastasis (BM) are usually viewed as in the terminal stage with a dismal prognosis. 
Recently, the incidence of BM in CRC patients was found to be increasing, although higher frequency screening and expanded management options for advanced CRC have improved patient survival. ${ }^{3,7}$ Many studies have been performed to explore clinic-pathologic and aberrant molecular features in CRC patients at high risk of developing BM.,7 In general, the prognosis of CRC patients with BM is very poor, with median overall survival (OS) ranging from 1 to 6 months, ${ }^{2,7-11}$ which is significantly shorter than that in patients with other types of primary malignancies. ${ }^{12}$ Accurate assessment of survival based on patient features will help in the design of tailored medical treatments for individual patients. Therefore, various potential factors have been reported to be associated with an improved patient prognosis, including fewer BM lesions, a better Karnofsky performance status (KPS), and the absence of extracranial metastases. ${ }^{2,7-11,13}$ However, there are inconsistencies with respect to the conclusions of these previous studies. For example, both good performance status and less BM were considered to be better prognostic predictors in the majority of previous studies, ${ }^{5,8,11,13}$ but they failed to correlate with OS in the analysis of patients treated surgically, ${ }^{14}$ or their significance regarding OS decreased when treatment modality was included. ${ }^{2,10}$

Apart from the selection bias and limited samples available from retrospective studies, therapeutic modality was another determining issue accounting for the heterogeneity in the published results. Increasing evidence suggests that aggressive treatment, including surgical resection and radiotherapy, could significantly extend survival outcomes in CRC patients with BM. ${ }^{13-15}$ In contrast, the majority of previous studies enrolled patients who had undergone both aggressive and palliative care. ${ }^{2,7-11}$ In light of the significant role of treatment modality, we performed survival analysis on the entire cohort of patients as well as on the subgroup of patients that was treated aggressively. Intriguingly, the results demonstrated a distinct profile of prognostic factors in this subgroup that underwent aggressive treatment. Furthermore, the presence of bone metastasis was demonstrated to independently predict a poor prognosis only in patients who received aggressive treatment.

\section{Patients and methods}

\section{Patients and data collection}

All patients treated at the Sun Yat-sen University Cancer Center were prospectively enrolled in a database. Each patient was followed up every 6 months until patient death or loss of medical record. Using this database, we identified 78 CRC patients diagnosed with BM between April 1991 and May
2017. The diagnosis of BM in CRC was confirmed using contrast-enhanced computerized tomography and/or magnetic resonance imaging, with or without pathological evidence. Among the 78 patients with BM, 51 patients received neurosurgical resection and/or radiotherapy to treat their BM. The last follow-up was in May 2017, which included verification of clinical attendance records and direct telecommunication with the patient or his/her family. Partial data from 60 patients, treated between April 1991 and December 2010, had been summarized in one of our previous studies [5].

Clinical information was retrospectively collected, including age, gender, site and Dukes' staging of primary CRC tumor, location of extracranial metastases, KPS, interval between the time of diagnosis of BM and CRC, the number and location of BM lesions, treatment modality of BM, and the maximum dimension of the BM. Patients were assigned to a Radiation Therapy Oncology Group recursive partitioning analysis (RPA) classification after the diagnosis of BM. The cause of death was also determined referring to a previously described protocol. ${ }^{16}$ Aggressive treatments included surgical resection, stereotactic radiosurgery (SRS), whole brain radiotherapy (WBRT), and/or chemotherapy. Cutoff time for the assignment of the metastasis as metachronous or synchronous was 30 days.

\section{Ethics approval and consent to participate}

All procedures performed in studies involving human participants were in accordance with the ethical standards of the Medical Ethics Committees of Sun Yat-sen University Cancer Center and followed the 1964 Helsinki Declaration and its later amendments or comparable ethical standards. The Medical Ethics Committees of Sun Yat-sen University Cancer Center approved this study. We also confirm that we have obtained written informed consent to review medical data and to participate in this study from all patients.

\section{Statistical analysis}

All statistical analyses were performed using SPSS software version 17.0 (SPSS Inc., Chicago, IL, USA). OS was defined as the time between the radiographic diagnosis of BM and death or until the date of the last follow-up visit. OS was estimated using the Kaplan-Meier method, and the statistical significance of the differences was assessed using the log-rank test. All significant parameters identified using univariate analysis were evaluated using multivariate analysis in the Cox proportional hazards model. The strength of the association between predictors and survival was assessed 
Table I Characteristics of primary colorectal carcinoma $(n=78)$

\begin{tabular}{ll}
\hline Characteristic & Patients, $\mathbf{n}(\%)$ \\
\hline Age at diagnosis of CRC & \\
Median (range), years & $56.8(2 \mathrm{I}-82)$ \\
Gender & \\
Female & $3 \mathrm{I}(39.7)$ \\
Male & $47(60.3)$ \\
Site of colorectal carcinoma & \\
Ascending colon & $12(15.4)$ \\
Transverse colon & $1 \mathrm{I}(14.1)$ \\
Descending colon & $2(2.7)$ \\
Sigmoid colon & $14(18.0)$ \\
Rectum & $38(48.7)$ \\
Multifocal primaries & $\mathrm{I}(1.3)$ \\
Dukes' staging & \\
A & $3(3.8)$ \\
B & $1 I(14.1)$ \\
C & $23(29.5)$ \\
D & $4 I(52.6)$ \\
\hline
\end{tabular}

Notes: Dukes' classification was used to assess the stage of primary CRC, where $A$ as invasion into but not through the bowel wall, $B$ invasion penetrating the muscle layer, $C$ involvement of lymph nodes, and D widespread metastases.

Abbreviation: CRC, colorectal cancer.

using hazard ratios (HRs) with 95\% CIs. A two-sided $P<0.05$ was considered statistically significant.

\section{Results}

\section{Clinical characteristics of primary CRC and $\mathrm{BM}$}

The clinical features of primary CRCs are summarized in Table 1. A total of $78 \mathrm{CRC}$ patients with BM were identified, comprising $47(60.3 \%)$ males. The median age at diagnosis of CRC was 56.8 years (range, $21-82$ years). Primary tumors were mostly located in the rectum (48.7\%) and sigmoid colon (18.0\%).

In Table 2, the clinical characteristics of the brain lesions are summarized. The median age at the time of diagnosis of BM was 58.7 years (range, 21-82 years). The median interval from the time of diagnosis of CRC to the development of BM was 23.2 months (range, 0-98 months). Thirteen (16.7\%) were synchronous BM. Patients had a predominantly $(\mathrm{n}=56$, $71.8 \%$ ) good KPS score of $\geq 70$. Nearly all patients $(n=68$, $87.2 \%$ ) had extracranial metastases at the time of diagnosis of BM. The most common sites of extracranial metastases were lung $(n=51,65.4 \%)$, followed by liver $(n=25,32.1 \%)$ and bone $(n=12,15.4 \%)$. Nearly half of the patients had single brain lesions $(37,47.4 \%)$, and the lesions were most frequently located in the supratentorial region $(n=44,56.4 \%)$.

\section{Treatment for BM and cause of death analysis}

Treatment modalities for patients with BM varied and depended on many factors, including general symptoms,
Table 2 Characteristics of brain metastasis $(n=78)$

\begin{tabular}{|c|c|}
\hline Characteristic & Patients, n(\%) \\
\hline \multicolumn{2}{|l|}{ Age at diagnosis of BM } \\
\hline Median (range), years & $58.7(21-82)$ \\
\hline$<65$ years & $42(53.8)$ \\
\hline$\geq 65$ years & $26(46.2)$ \\
\hline \multicolumn{2}{|c|}{ Site of extracranial metastases } \\
\hline Lung & $51(65.4)$ \\
\hline Liver & $25(32.1)$ \\
\hline Bone & $12(15.4)$ \\
\hline Other sites & $24(30.8)$ \\
\hline \multicolumn{2}{|l|}{ Diagnostic methods } \\
\hline СT/PET-CT & $32(41.0)$ \\
\hline MRI & $46(59.0)$ \\
\hline \multicolumn{2}{|c|}{ Location of brain lesions } \\
\hline Supratentorial & $44(56.4)$ \\
\hline Infratentorial & $16(20.5)$ \\
\hline Combinations & $18(23.1)$ \\
\hline \multicolumn{2}{|c|}{ Interval between diagnosis of BM and CRC } \\
\hline Median (range), months & $23.17(0-98)$ \\
\hline$<24$ months & $45(57.7)$ \\
\hline$\geq 24$ months & $33(42.7)$ \\
\hline \multicolumn{2}{|c|}{ Diameter of the largest BM } \\
\hline Median (range), mm & $27.7(I-47)$ \\
\hline$<30 \mathrm{~mm}$ & $47(60.3)$ \\
\hline$\geq 30 \mathrm{~mm}$ & $31(39.7)$ \\
\hline \multicolumn{2}{|c|}{ Number of brain lesions } \\
\hline 1 & $37(47.4)$ \\
\hline 2 & $9(I I .5)$ \\
\hline$\geq 3$ & $32(4 I . I)$ \\
\hline \multicolumn{2}{|l|}{ KPS } \\
\hline$\geq 70$ & $56(71.8)$ \\
\hline$<70$ & $22(28.2)$ \\
\hline \multicolumn{2}{|l|}{ RPA class } \\
\hline I & $7(9.0)$ \\
\hline II & $49(62.8)$ \\
\hline III & $22(28.2)$ \\
\hline
\end{tabular}

Abbreviations: BM, brain metastasis; CRC, colorectal cancer; KPS, Karnofsky performance status; MRI, magnetic resonance imaging; PET-CT, positron emission tomography-computed tomography; RPA, recursive partitioning analysis.

Table 3 Treatment modalities and sequences $(n=78)$

\begin{tabular}{ll}
\hline Sequence of treatments & Patients, n (\%) \\
\hline Surgery only & $4(5.1)$ \\
Surgery + chemotherapy & $5(6.4)$ \\
Surgery - WBRT + SRS + chemotherapy & $2(2.6)$ \\
Surgery-SRS + chemotherapy & $2(2.6)$ \\
WBRT only & $7(9.0)$ \\
WBRT + chemotherapy & $4(5.1)$ \\
WBRT + chemotherapy - Surgery & $3(3.8)$ \\
SRS only & $5(6.4)$ \\
SRS + chemotherapy & $6(7.7)$ \\
SRS + WBRT & $5(6.4)$ \\
SRS + WBRT + chemotherapy & $8(10.3)$ \\
Steroid only & $27(34.6)$
\end{tabular}

Abbreviations: SRS, stereotactic radiosurgery; WBRT, whole brain radiotherapy 
extent of disease, number and location of brain lesions, patient wishes, and physician discretion. Treatment information is shown in Table 3. Sixteen (20.5\%) of the patients underwent surgical resection, and thirty-five (44.9\%) patients received only radiotherapy. The remaining 27 (34.6\%) were given palliative treatment only. Twenty-nine patients received systemic chemotherapy after treatment of BM. Chemotherapy protocols were mainly composed of irinotecan, 5-FU, or oxaliplatin $(\mathrm{n}=24)$, and five patients were treated with bevacizumab. Only two patients with bone metastases received systemic chemotherapy. Additionally,

Table 4 Cause of death $(n=78)$

\begin{tabular}{llll}
\hline $\begin{array}{l}\text { Treatment } \\
\text { modality }\end{array}$ & $\begin{array}{l}\text { Steroid } \\
\text { only }\end{array}$ & $\begin{array}{l}\text { Surgery or } \\
\text { SRS or } \\
\text { WBRT only }\end{array}$ & $\begin{array}{l}\text { WBRT and } \\
\text { SRS or } \\
\text { surgery }\end{array}$ \\
\hline $\begin{array}{l}\text { Patients alive at } \\
\text { the last follow-up }\end{array}$ & 0 & 2 & $\mathrm{I}$ \\
$\begin{array}{l}\text { Cause of death } \\
\text { Neurological }\end{array}$ & 17 & $\mathrm{II}$ & 5 \\
$\begin{array}{l}\text { Systemic } \\
\text { Both }\end{array}$ & 6 & 8 & $\mathrm{II}$ \\
\hline Ab & 3 & $\mathrm{II}$ & 3 \\
\hline
\end{tabular}

Abbreviations: SRS, stereotactic radiosurgery; WBRT, whole brain radiotherapy six patients were cured with brain lesion-targeted chemotherapy regimens, containing either cisplatin, tiniposide, nimustine, or temozolomide.

At the end of follow-up, three patients were still alive. As shown in Table 4, neurological failure accounted for $65.4 \%$ of deaths in patients who received steroid only. In contrast, only $25 \%$ of patients who received multiple treatments died of neurological failure. Among the patients with bone metastases at the diagnosis of BM, six of them died of systemic progression, three of neurological failure, and three of both systemic and neurological progression.

\section{Survival and prognostic analysis in the entire patient cohort}

The median OS of all the patients was 7.0 months (95\% CI: 4.6-9.4 months) from the time of diagnosis of BM (Figure 1A). OS at 6 months, 12 months, and 24 months was estimated to be $55.1 \%, 32.5 \%$, and $9.5 \%$, respectively.

Univariate and multivariate analyses were performed to evaluate prognostic factors that included age at the time of diagnosis of BM; interval between the time of diagnosis
A

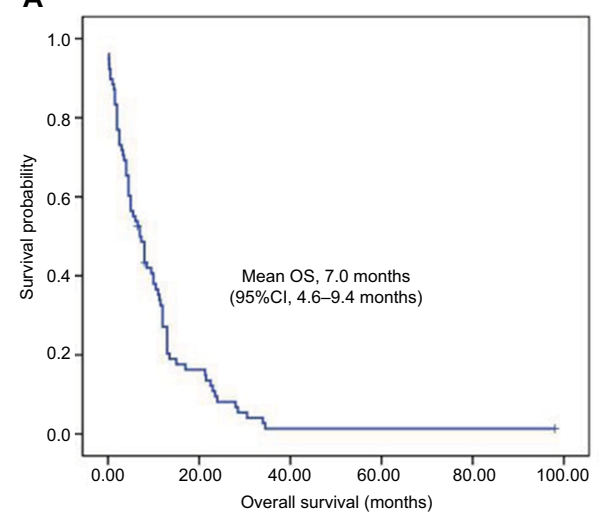

D

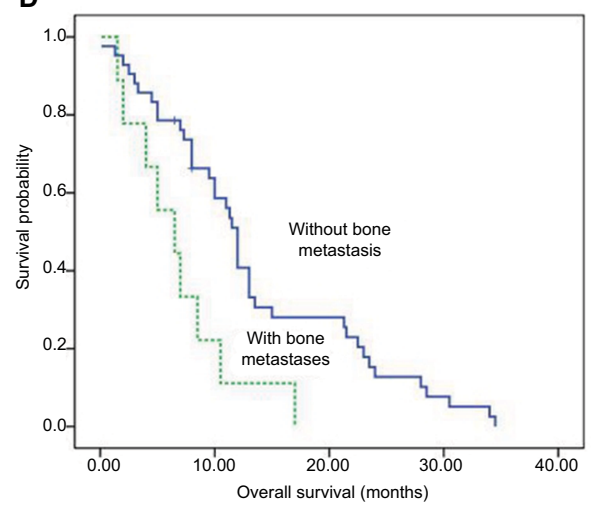

B

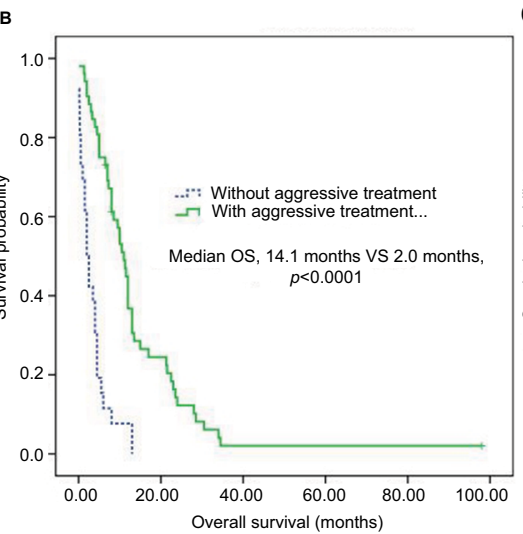

E

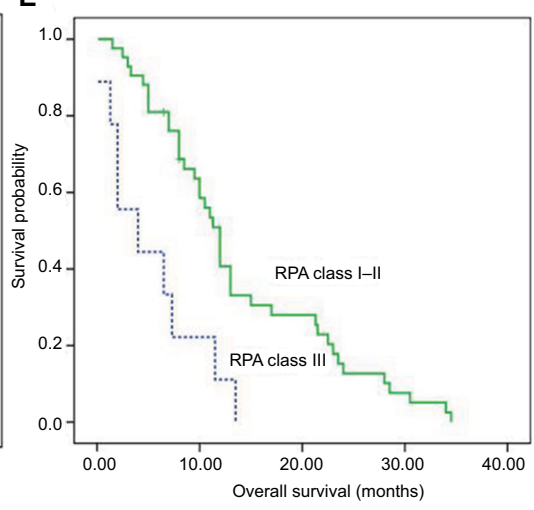

C

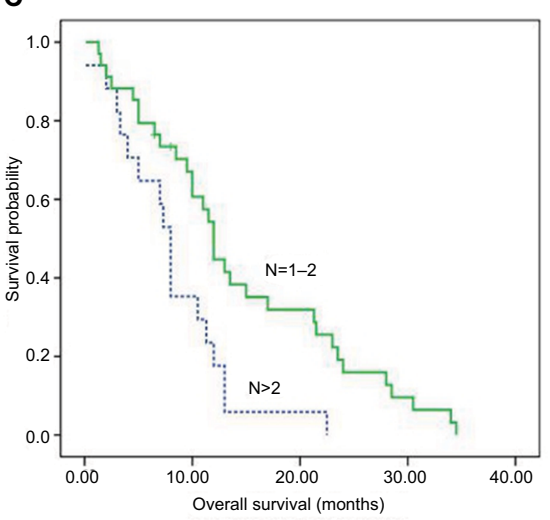

Figure I (A) Kaplan-Meier analysis of OS in 78 patients, (B) according to treatment modality $(n=78),(\mathbf{C})$ the number of brain metastases ( $n=5 \mathrm{I}$ ), (D) the presence of bone metastases $(n=5 I)$, and (E) RPA class $(n=5 I)$.

Abbreviations: OS, overall survival; RPA, recursive partitioning analysis. 
of BM and CRC; location; diameter and number of brain lesions; presence of liver, lung, or bone metastasis; and RPA class. Among them, the number of BM, location of $\mathrm{BM}$, age, and RPA class were demonstrated to be associated with OS in univariate analysis (Table 5). However, only RPA class I/II $(\mathrm{HR}=0.31,95 \% \mathrm{CI}: 0.18-0.53, P<0.0001)$

Table 5 Univariate analysis of survival $(n=78)$

\begin{tabular}{|c|c|c|c|c|}
\hline Variable & $\mathbf{n}$ & $\begin{array}{l}\text { Median OS } \\
\text { (months) }\end{array}$ & $\begin{array}{l}\text { Mean OS } \\
\text { (months) }\end{array}$ & P-value \\
\hline Overall survival & 78 & 7.00 & 10.50 & \\
\hline \multicolumn{4}{|c|}{ Age at the diagnosis of brain metastasis } & 0.028 \\
\hline$<65$ years & 42 & 10.00 & 13.90 & \\
\hline$\geq 65$ years & 36 & 4.00 & 7.66 & \\
\hline Number of brain & & & & 0.013 \\
\hline \multicolumn{5}{|l|}{ metastasis } \\
\hline $\mathrm{I}-2$ & 46 & 10.00 & 11.90 & \\
\hline$\geq 3$ & 32 & 4.00 & 8.40 & \\
\hline \multicolumn{4}{|c|}{ Location of brain metastasis } & 0.019 \\
\hline Supratentorial & 44 & 9.50 & 12.50 & \\
\hline Infratentorial & 16 & 8.00 & 10.60 & \\
\hline Both & 18 & 4.00 & 5.60 & \\
\hline RPA class & & & & 0.0001 \\
\hline $\mathrm{I} / \mathrm{II}$ & 56 & 10.00 & 13.20 & \\
\hline III & 22 & 2.00 & 3.80 & \\
\hline
\end{tabular}

Abbreviations: OS, overall survival; RPA, recursive partitioning analysis. and the number of $\mathrm{BM}(\mathrm{HR}=0.55,95 \% \mathrm{CI}: 0.34-0.89$, $P=0.014)$ were shown to independently predict survival in the multivariate model.

\section{Survival and prognostic analysis in patients undergoing aggressive treatment}

Among the 78 patients, 51 were treated aggressively, and their OS was significantly longer than that in patients who accepted palliative care (14.1 months vs 2.0 months, $P<0.0001$; Figure 1B). OS in this aggressively treated patient cohort at 6,12 , and 24 months was estimated to be $74.5 \%$, $43.8 \%$, and $12.5 \%$, respectively. Three of them remained alive at the last follow-up.

Similarly, univariate and multivariate analyses were performed to evaluate prognostic factors. In univariate analysis, the number of BM (Figure 1C), location of BM, bone metastasis at the time of diagnosis of BM (Figure 1D), and RPA class (Figure 1E) were demonstrated to be associated with OS (Table 6). Interestingly, the presence of bone metastasis $(\mathrm{HR}=2.38,95 \% \mathrm{CI}: 1.08-5.28, P=0.032)$, rather than lung or liver metastasis, was demonstrated to predict a poor prognosis, independent of RPA class I/II and the number of BM in the multivariate model.

Table 6 Univariate and multivariate analysis of survival $(n=5 \mathrm{I})$

\begin{tabular}{|c|c|c|c|c|c|c|}
\hline \multirow[t]{2}{*}{ Variable } & \multirow[t]{2}{*}{$\mathbf{n}$} & \multirow[t]{2}{*}{ Median OS (months) } & \multirow{2}{*}{$\begin{array}{l}\text { Univariate analysis } \\
P \text {-value }\end{array}$} & \multicolumn{3}{|c|}{ Multivariate analysis } \\
\hline & & & & $\overline{\text { HR }}$ & $95 \% \mathrm{Cl}$ & $P$-value \\
\hline Overall survival & 51 & 11 & & & & \\
\hline \multicolumn{3}{|c|}{ Age at the diagnosis of BM } & 0.107 & & & \\
\hline$<65$ years & 33 & 11 & & & & \\
\hline$\geq 65$ years & 18 & 7.3 & & & & \\
\hline \multicolumn{7}{|c|}{ Lung metastases } \\
\hline Yes & 37 & 10.5 & 0.759 & & & \\
\hline No & 14 & 11.3 & & & & \\
\hline \multicolumn{7}{|c|}{ Liver metastases } \\
\hline Yes & 16 & 8.0 & 0.349 & & & \\
\hline No & 35 & 11.5 & & & & \\
\hline Bone metastase & & & 0.006 & 2.38 & $1.08-5.28$ & 0.032 \\
\hline Yes & 9 & 6.5 & & & & \\
\hline No & 42 & 12 & & & & \\
\hline Number of BM & & & 0.005 & 0.4 & $0.21-0.78$ & 0.007 \\
\hline $\mathrm{I}-2$ & 34 & 12 & & & & \\
\hline$\geq 3$ & 17 & 8 & & & & \\
\hline Location of BM & & & 0.045 & & & \\
\hline Supratentorial & 29 & 12 & & & & \\
\hline Infratentorial & 12 & 8 & & & & \\
\hline Both & 10 & 7 & & & & \\
\hline RPA class & & & 0.001 & 0.27 & $0.12-0.6$ & 0.001 \\
\hline I/II & 42 & 12 & & & & \\
\hline III & 9 & 4 & & & & \\
\hline
\end{tabular}

Abbreviations: BM, brain metastasis; HR, hazard ratio; OS, overall survival; RPA, recursive partitioning analysis. 


\section{Discussion}

$\mathrm{BM}$ in CRC was considered a rare event, with a very poor median OS (range, 1-6 months). ${ }^{2,7-11}$ With the advent of substantial improvements in the management of advanced CRC, the incidence of BM appears to be increasing. ${ }^{3,7}$ Various studies have been performed to explore potential prognostic factors to define subgroups of patients that might benefit from aggressive treatment. However, the profile of prognostic factors remains undefined due to the inconsistency of previous studies. Since treatment modality is the major determinant of patient survival, we hypothesized that the pattern of prognostic factors between patients treated aggressively and palliatively is different. In the present study, we conducted survival analysis on the entire cohort of patients and the subsection treated aggressively. Surprisingly, the results demonstrated a distinct profile of prognostic factors in the subgroup of patients that underwent aggressive treatment. Similar to previous findings, multiple BMs and worse performance status were associated with a poorer prognosis either in the entire cohort of patients or in patients treated aggressively. However, the presence of bone metastasis was demonstrated to independently predict a poor prognosis only in patients who accepted aggressive treatment. To our knowledge, this is the first study to reveal a predictive role of bone metastasis in advanced CRC patients with BM. With progression of brain disease, the neurological status of patients is expected to decline. Subsequently, any treatment modality that would extend survival even by a few weeks while providing a reasonable quality of life in the terminal stages would be significant. Several studies have provided evidence that has shown that aggressive treatment for the BM, including neurosurgical resection, SRS, and WBRT, was able to significantly prolong OS in CRC patients with BM., ${ }^{2,10,14,15}$ Similarly, patients who received aggressive treatment for their BM survived much longer than those who received only palliative care ( 14.1 months vs 2.0 months, $P<0.0001$ ). Furthermore, neurological failure was the main cause of death in patients who received only steroids, while patients who received aggressive treatment died mainly of systemic disease. This indicated that controlling brain lesions helped to decrease BM-induced death.

However, it remains very difficult to formulate an optimal strategy to treat BM based on the pretreatment features of patients. Although various studies have been performed to try to define the subgroup of patients who might benefit from aggressive treatment, the identified prognostic factors were inconsistent among different studies. In the majority of previous studies, good performance status and fewer BM were considered better predictors of prognosis. ${ }^{5,8,11,13}$ Yet, both of these factors failed to correlate with OS in the analysis of patients treated surgically, ${ }^{14}$ or the significance of these factors regarding survival decreased when incorporated with treatment modality. ${ }^{2,10}$ To avoid the influence of treatment modality, we performed survival analysis on the entire cohort of patients, as well as on those who underwent aggressive treatment. In both cohorts, RPA class and the number of BM were demonstrated to independently predict patient survival. Patients with a limited number of BM and better performance status tended to receive aggressive treatment, including surgical resection, radiotherapy, and the combination of them, which has been shown to improve prognosis., ${ }^{3,9,14,17}$ Our study provides further convincing evidence to support the role of the number of $\mathrm{BM}$ and performance status in determining treatment strategy.

Extracranial metastases, reflecting the burden of systemic diseases, were commonly regarded as a prognostic factor of poor outcomes in CRC patients with BM.,7 However, this has not always been the case in some studies, where extracranial metastases were not statistically significantly associated with OS. ${ }^{5,10,11}$ The overwhelming proportion of extracranial metastases in the majority of the study groups partially accounts for this discrepancy. According to previous studies, metastatic CRC exhibited a sequential progression in many patients, with metastasis usually seen earliest in lung, followed by liver and bone. ${ }^{4,5,10,11}$ In other words, the presence of metastasis in each site may indicate a gradual increasing extension of systemic burden for patients with CRC. Therefore, the involvement of different extracranial organs should affect OS in a prognostically distinct manner in CRC patients with BM. Thus, we explored the effect of lung, liver, and bone metastasis on OS individually. In the entire cohort of patients, none of them were significantly correlated with OS. In contrast, when evaluated in the patients treated aggressively, the presence of bone metastasis, rather than lung or liver, independently predicted a poor prognosis. In support of our results, the presence of lung metastasis did not affect OS in previous studies, ${ }^{3,14}$ but could predict the emergence of BM. ${ }^{3}$ Therefore, even in CRC patients with the accompanying presence of metastasis to liver and lung, aggressive management of their diseases remains indicated for patients with BM. As far as we know, this study was the first to reveal the significance of bone metastasis in CRC patients with BM, which could aid in evaluating whether patients with BM will benefit from aggressive treatment. 
In the majority of previous studies, median OS was very poor, ranging from 1 to 6 months., ${ }^{2,-11,13}$ Through the use of multidisciplinary disease management and aggressive treatment, patient survival is improving. ${ }^{4,5,14,17}$ As reported previously, the median OS of all patients in the present study was 7.0 months. The OS of patients treated aggressively was dramatically longer than the OS in those treated conservatively ( 14.1 months vs 2.0 months, $P<0.0001$ ); this confirms that the nature of the treatment modality influences the prognosis of CRC patients with BM.

The significance of the present study was limited by its retrospective design, selection bias, and physician discretion. Furthermore, we unfortunately did not have access to the information on RAS gene mutations, which might be associated with worse survival and an increase in metastatic CRC. ${ }^{6}$ In addition, treatment modality varied among patients, and some of them did not follow the standard treatment strategy, perhaps given its respective design and patient choice. Nevertheless, the present study demonstrated a distinct profile of prognostic factors by performing survival analysis on the entire cohort of patients as well as those who underwent aggressive treatment. Finally, the patients with bone metastasis had relatively poor prognosis compared with those without, is a preliminary but interesting finding. The small number of patients with bone metastases limited the validity of the conclusion. Further studies with larger cases are warranted to validate the efficacy of bone metastasis in predicting the prognosis of patients with $\mathrm{BM}$ from CRC.

\section{Conclusion}

Although the presence of $\mathrm{BM}$ is usually considered a terminal-stage phenomenon of CRC, a subgroup of patients with few brain lesions and good performance status could have a satisfactory survival time after aggressive treatment. However, those with bone metastasis at the time of diagnosis of their BM had a much worse prognosis than those without. Detailed evaluation of the systemic tumor burden helps to accurately assess CRC patients with BM.

\section{Acknowledgments}

Data from some of the patients used in this study had been retrospectively summarized in our previous study. ${ }^{5}$ The abstract of the present study was accepted as an oral presentation in Asian society for neuro-oncology ASNO 2017.

We thank Mark Abramovitz, PhD, from Liwenbianji, Edanz Group China (www.liwenbianji.cn/ac), for editing the English text of a draft of this manuscript.
This work was supported by the National Natural Science Foundation of China for Young Scholars (81702479) and the Medical Scientific Research Foundation of Guangdong Province (A2017188), Doctoral Program of Natural Science Foundation of Guangdong Province (2017A030310201), Science and Technology Planning Project of Guangdong Province, China (2015B010125003), and Science and Technology Program of Guangzhou, China (201704020133).

\section{Author contributions}

$\mathrm{HD}, \mathrm{ZQH}, \mathrm{XBJ}$, and YGM are responsible for the conception and design of this paper. JW, ZPC, and YGM are responsible for the administrative support. $\mathrm{HD}, \mathrm{ZQH}, \mathrm{CCG}$, and JHL are responsible for the collection of data. $\mathrm{HD}, \mathrm{ZQH}, \mathrm{KS}$, and XBJ are responsible for data analysis and interpretation. The writing of the manuscript was done by $\mathrm{HD}, \mathrm{ZQH}, \mathrm{ZZ}$, and XBJ. All authors contributed toward data analysis, drafting and revising the paper and agree to be accountable for all aspects of the work.

\section{Disclosure}

The authors report no conflicts of interest in this work.

\section{References}

1. Torre LA, Bray F, Siege 1RL, et al. Global cancer statistics, 2012. CA Cancer J Clin. 2015;65(2):87-108.

2. Damiens K, Ayoub JP, Lemieux B, et al. Clinical features and course of brain metastases in colorectal cancer: an experience from a single institution. Curr Oncol. 2012;19(5):254-258.

3. Nozawa H, Ishihara S, Kawai K, et al. Brain metastasis from colorectal cancer: predictors and treatment outcomes. Oncology. 2017;93(5):309-314.

4. Gu XD, Cai YT, Zhou YM, et al. Prognostic factors and multidisciplinary treatment modalities for brain metastases from colorectal cancer: analysis of 93 patients. BMC Cancer. 2015;15:902.

5. Jiang XB, Yang QY, Sai K, et al. Brain metastases from colorectal carcinoma: a description of 60 cases in a single Chinese cancer center. Tumour Biol. 2011;32(6):1249-1256.

6. Yaeger R, Cowell E, Chou JF, et al. RAS mutations affect pattern of metastatic spread and increase propensity for brain metastasis in colorectal cancer. Cancer. 2015;121(8):1195-1203.

7. Michl M, Thurmaier J, Schubert-Fritschle G, et al. Brain metastasis in colorectal cancer patients: survival and analysis of prognostic factors. Clin Colorectal Cancer. 2015;14(4):281-290.

8. Tokoro T, Okuno K, Hida JC, et al. Prognostic factors for patients with advanced colorectal cancer and symptomatic brain metastases. Clin Colorectal Cancer. 2014;13(4):226-231.

9. Fountzilas C, Chang K, Hernandez B, et al. Clinical characteristics and treatment outcomes of patients with colorectal cancer who develop brain metastasis: a single institution experience. J Gastrointest Oncol. 2017;8(1):55-63.

10. Kye BH, Kim HJ, Kang WK, et al. Brain metastases from colorectal cancer: the role of surgical resection in selected patients. Colorectal Dis. 2012;14(7):e378-e385.

11. Jung M, Ahn JB, Chang JH, et al. Brain metastases from colorectal carcinoma: prognostic factors and outcome. J Neurooncol. 2011;101(1):49-55. 
12. Fabi A, Felici A, Metro G, et al. Brain metastases from solid tumors: disease outcome according to type of treatment and therapeutic resources of the treating center. J Exp Clin Cancer Res. 2011;30:10.

13. Suzuki Y, Yamaguchi T, Matsumoto H, et al. Prognostic factors and treatment effects in patients with curatively resected brain metastasis from colorectal cancer. Dis Colon Rectum. 2014;57(1): 56-63.

14. Wroński M, Arbit E. Resection of brain metastases from colorectal carcinoma in 73 patients. Cancer. 1999;85(8):1677-1685.
15. Kim HJ, Huh JW, Jung TY, et al. Clinical outcome with gamma-knife surgery or surgery for brain metastases from colorectal cancer. J Clin Neurosci. 2013;20(10):1417-1421.

16. Patchell RA, Tibbs PA, Walsh JW, et al. A randomized trial of surgery in the treatment of single metastases to the brain. $N$ Engl J Med. 1990;322(8):494-500.

17. Paix A, Antoni D, Adeduntan R, Noël G. Stereotactic radiation therapy of brain metastases from colorectal cancer: a single institution cohort. Cancer Radiother. 2017;21(3):199-204.

\section{Publish your work in this journal}

Cancer Management and Research is an international, peer-reviewed open access journal focusing on cancer research and the optimal use of preventative and integrated treatment interventions to achieve improved outcomes, enhanced survival and quality of life for the cancer patient. The manuscript management system is completely online and includes a very quick and fair peer-review system, which is all easy to use. Visit http://www.dovepress.com/testimonials.php to read real quotes from published authors. 